\title{
Nom de genre et institutionnalisation d'une pratique discursive : le cas de l'interpellation parlementaire et du signalement d'enfant en danger
}

\author{
Caroline Mellet \\ caroline.mellet@wanadoo.fr \\ Université Paris Ouest Nanterre La Défense \& MoDyCo \\ Frédérique Sitri \\ fsitri@u-paris10.fr \\ Université Paris Ouest Nanterre La Défense \& MoDyCo
}

\begin{abstract}
Notre contribution a pour objectif de réfléchir à une approche de la notion de genre de discours qui rende compte de son historicité. Cette caractéristique, soulignée par Bakhtine, constitue une conséquence immédiate de son approche du genre comme production discursive en interaction étroite avec la situation qui en permet l'irruption. Notre étude propose de cerner au plus près les processus d'émergence et d'institutionnalisation de deux genres de discours historiquement situés et appartenant à deux sphères d'activité différentes : le signalement d'enfant en danger et l'interpellation parlementaire. Cette analyse comparative nous permettra d'envisager une définition renouvelée du genre.
\end{abstract}

Nous situons tout d'abord notre étude parmi les différentes recherches sur les genres de discours, avant de rendre compte des interactions complexes et dynamiques entre nom de genre, but pragmatique et formes linguistiques. La dernière partie de notre contribution porte sur l'analyse des processus d'émergence du signalement d'enfant en danger, au sein de la sphère du travail social et de l'interpellation parlementaire, dans la sphère politique.

\section{Les études sur les genres de discours}

On assiste depuis plusieurs années à un intérêt renouvelé pour la notion de genre en analyse du discours. Les propositions bakhtiniennes, sous-tendues par une critique à l'égard de la conception de la parole chez Saussure ${ }^{1}$, ont suscité de nombreuses études en dehors de la sphère littéraire où cette notion a longtemps été cantonnée. Les raisons de ce renouveau, en particulier pour les genres ordinaires, ont été analysées par Moirand (2003). Pourtant, même si quelques travaux témoignent d'une réflexion sur la définition du genre $^{2}$, la plupart d'entre eux s'appuient sur une définition intuitive de cette notion ou constituent des corpus à partir de catégories génériques identifiées par un nom de genre tenu pour acquis ${ }^{3}$. Les critères permettant de rassembler ou au contraire de séparer des textes en fonction de la catégorie générique ne sont pas toujours suffisamment interrogés. Il en est ainsi, par exemple de l'appréhension des genres chez Rastier :

Or le corpus de textes du même genre s'impose en général: on juge d'une délibération de conseil d'administration par rapport aux précédentes, d'un poème par rapport à d'autres poèmes et non par rapport à des romans. (2001 : 232)

$\mathrm{Si}$, selon lui, le corpus de textes « s'impose », c'est, semble-t-il, parce qu'il considère que le nom de genre suffit à sa délimitation. Plusieurs auteurs (dont par exemple Moirand, 2003) réfléchissent aux catégories pertinentes pour l'identification et la classification des genres et certains soulignent les problèmes de reconnaissance des genres, mais trop peu d'études interrogent la relation entre genre et nom de genre. Certes Maingueneau (2004a, 2009) et Bouquet (2007) soulignent la nécessaire prise en compte du nom de genre dans l'interprétation, et Bronckart remarque même avec beaucoup de justesse : 


\begin{abstract}
Les frontières entre genres ne peuvent pas toujours être clairement établies (cf le problème des frontières entre roman et nouvelle, ou entre article scientifique et article didactique, etc.), et (que) certaines sortes de textes nouvelles peuvent ne pas encore avoir reçu d'appellation consacrée en terme de genre. De sorte que l'organisation des genres se présente aux yeux des usagers d'une langue sous forme d'une nébuleuse, comportant des îlots plus ou moins stabilisés (genres clairement définissables et étiquetables), et des ensembles de textes aux contours flous et en intersection partielle (genres pour lesquels les définitions et les critères de classements restent mobiles et/ ou divergents). (1997: 76)
\end{abstract}

S'appuyant sur une conception dynamique des genres, Bronckart met particulièrement en évidence la difficulté d'identification des genres en émergence dans une sphère d'activité ; il remarque, mais sans s'y attarder, l'importance de la nomination dans le processus de reconnaissance des genres par la communauté discursive. Néanmoins, ne sont interrogées ni les difficultés liées à la coexistence de noms de genres proches dans une sphère donnée, ni même les processus d'émergence du nom, ou encore les distorsions entre le nom donné à un genre par une institution et la réalité pragmatique à laquelle ce nom renvoie. On citera encore l'étude de Dolinine (1999), qui, appréhendant les genres de discours comme déterminés par la situation de communication dans laquelle ils sont produits, consacre une part de sa réflexion à la question des «noms» de genre, et tout particulièrement à la question de la relation entre « acte de langage » et « nom de genre»

Enfin, la dimension historique des genres est encore trop peu étudiée. Remarquée par Bakhtine, elle est soulignée par Bronckart ${ }^{4}$ ou encore par Moirand (2003). Adam (1999: 87) note même la nécessité d'analyses prenant en compte la dimension diachronique des genres de discours: «Il me semble indispensable d'étudier la spécificité et l'historicité des genres du discours publicitaire, des genres de la presse écrite ou des genres littéraires ». Pourtant peu d'études, intégrant des corpus diachroniques, concernent les processus d'émergence et d'institutionnalisation de genres de discours au sein d'une sphère d'activité particulière'.

L'objectif de notre contribution est justement de repenser la notion de genre en montrant le caractère déterminant de la dimension diachronique. L'étude des processus d'émergence et d'institutionnalisation des genres de discours met en évidence les interactions complexes et dynamiques entre nom de genre, visée pragmatique et formes linguistiques.

\title{
2 Une appréhension dynamique du genre : l'interaction entre nom de genre, visée pragmatique et formes linguistiques
}

\subsection{Les distorsions entre nom, visée pragmatique et formes linguistiques}

Il convient tout d'abord d'instaurer une distinction théorique entre les noms des genres de discours, leur visée pragmatique réelle et les formes linguistiques qui peuvent leur être associées. Même si ces différentes composantes peuvent contribuer à la reconnaissance et la définition des genres, des distorsions importantes peuvent en effet exister entre ces éléments. Non seulement l'appellation peut ne pas correspondre à la visée pragmatique réelle ${ }^{6}$, mais inversement, le même but pragmatique et des caractéristiques linguistiques voisines peuvent recevoir des appellations diverses, en fonction de la communauté discursive qui l'évalue ou même de l'institution qui peut hésiter, en fonction des intentions qu'elle se donne, entre plusieurs noms ${ }^{7}$. En outre, le genre, en passant d'une sphère d'activité à une autre, peut garder un nom identique tout en subissant des transformations si importantes qu'il peut être légitime d'interroger son identité. Encore faut-il que cette interrogation se fonde sur des études spécifiques à l'aide de critères précis pour chaque sphère. Rastier, prenant l'exemple de la lettre, affirme que le changement de sphère d'activité entraîne un changement de genre :

Comme les genres restent subordonnés à des discours, l'existence de genres transdiscursifs reste douteuse, car le voisinage d'autres genres, ou s'il s'agit de genres inclus, d'autres contextes d'inclusion, suffit à les modifier : un proverbe, par exemple, 


\begin{abstract}
n'a pas le même sens dans un discours ludique ou dans un discours juridique ; la lettre commerciale n'a presque rien à voir avec la lettre personnelle du discours privé, car la corrélation entre contenu et expression demeure critériale pour définir le genre. (2001, 253)
\end{abstract}

Or, un tel jugement ne nous semble pas pouvoir être appliqué sans discernement à l'ensemble des genres discursifs. Le critère du contenu n'est pas suffisant et celui de l'expression nous semble trop vague. Des analyses comparatives détaillées doivent être menées de manière systématique.

Sans prétendre à une analyse complète des différentes configurations entrant en jeu entre ces éléments, nous porterons plus particulièrement notre attention sur les enjeux liés aux dénominations des genres.

\title{
2.2 Approche d'une catégorie de noms de genres : les déverbaux
}

Une entrée possible dans l'étude des relations dynamiques pouvant permettre l'identification des genres, consiste en effet à s'intéresser à leur dénomination. Petit et Beacco (2004), étudiant ainsi les rapports entre noms de dire et genres de discours, distinguent deux catégories de noms pouvant se rapporter à un genre discursif: les noms de dire non-dérivés (livre, article, post-it, etc.), dénommant en général un support ou une production marquée par ses conditions de production, et les déverbaux de noms de dire. Nous réservant la possibilité d'étudier dans des travaux ultérieurs les noms liés à la première catégorie ${ }^{8}$, nous porterons notre attention sur les noms de genre liés à des dérivés de verbes de dire, parmi lesquels peuvent être isolés les verbes employés comme performatifs explicites. Ces verbes, manifestant une capacité des locuteurs à catégoriser l'activité discursive, témoignent d'une compétence « métalinguistique ordinaire ", pour reprendre les termes utilisés par Petit et Beacco, permettant dans certains cas l'émergence de noms de genre. Les auteurs soulignent les valeurs polysémiques de ces déverbaux, puisque ceux-ci peuvent désigner aussi bien une valeur processuelle (acte de discours) qu'une valeur résultative (forme discursive). Cette remarque est confirmée par les analyses menées sur les corpus que nous étudions: globalement, il apparaît nécessaire de découpler les référents des noms pouvant correspondre à un genre. Ces noms peuvent en effet renvoyer au moins à trois référents :

- Le déverbal peut avoir une valeur processuelle et désigner 1a nominalisation de l'acte performatif: l'interpellation ou le signalement réfèrent alors à l'acte de dire « j’interpelle », « je signale ».

- Le déverbal peut également correspondre à l'activité discursive d'interpellation ou de signalement. Le nom désigne alors l'activité discursive qui a pour but pragmatique d'interpeller ou de signaler. Cette activité ne correspond pas forcément au nom du genre mais à une évaluation, par le locuteur, l'interlocuteur, ou encore un tiers évaluateur de ce que le locuteur fait ou a fait en parlant. Dans le cas de « signalement », par exemple, nous verrons que les locuteurs qualifient de «signalement» un texte qui porte un nom de genre institutionnel différent (« information préoccupante » ou « rapport »).

- Enfin, le déverbal peut désigner un nom de genre institué (une interpellation parlementaire, un signalement d'enfant en danger), correspondant à des normes discursives stabilisées.

Ainsi peuvent s'expliquer les distorsions éventuelles entre le nom et le but pragmatique, et même le performatif explicite ou l'acte. Les référents étant parfois distincts, il convient par exemple de ne pas établir d'association systématique entre un nom de genre désignant la nomination d'un acte performatif et le but pragmatique réel. On peut aussi supposer que l'institutionnalisation d'un genre peut contribuer à l'éloigner de l'acte performatif ou de l'activité discursive qu'il a pu à un moment désigner. Par exemple, dans le cas du signalement, le genre institué comporte une dimension d'évaluation de la situation et de préconisation, dimension que n'intègre pas le simple acte de signalement. De la même façon, dans la sphère parlementaire, le genre des questions au Gouvernement comporte très fréquemment une phrase interrogative mais celle-ci ne correspond que très rarement à l'acte d'interrogation.

L'étude du ou des processus d'émergence de deux genres de discours historiquement situés et appartenant à des sphères d'activité distinctes permet de mettre en évidence certains de ces découplages. L'observation la plus précise possible de l'apparition d'un genre de discours met en effet en jeu les trois référents évoqués plus haut : l'activité discursive, en adéquation avec les conditions socio-historiques qui 
la rendent possible, peut intégrer le performatif explicite et s'accompagner d'une dénomination qui s'institue dans certains cas en genre de discours. C'est ce que nous nous proposons d'étudier.

\section{Etude comparée de l'émergence et de l'institutionnalisation de deux genres de discours : l'interpellation parlementaire et le signalement d'enfant en danger}

\subsection{Les corpus}

Nous travaillons à partir de deux corpus appartenant à deux sphères différentes. Etant donné nos questions de recherche, ces corpus ont des caractéristiques communes : il s'agit de corpus écrits, revêtant une dimension diachronique, et qui en l'état actuel de nos investigations ont une dimension exploratoire; dans le cas des écrits de signalement, l'accès aux archives (à condition même que tous les écrits soient archivés) est restreint par la non-communication des documents portant sur des individus ${ }^{9}$; pour ce qui est de l'interpellation, nous relevons deux difficultés, inhérentes à tout travail sur des archives en histoire : d'une part, les documents analysés constituent toujours une représentation de l'événement ${ }^{10}$ (en l'occurrence, des débats parlementaires). D'autre part, nous travaillons sur des événements révolus, toujours difficiles à contextualiser. Les conclusions présentées ici seront donc étayées ultérieurement par une exploration plus systématique et par l'élargissement du corpus.

\subsubsection{Le signalement d'enfant en danger}

Le corpus utilisé pour la présente communication est extrait d'un corpus plus large constitué autour de rapports éducatifs concernant des enfants « signalés » à la justice (voir Cislaru et al., 2008). Quand le cas d'un enfant en danger ou en risque de danger est porté à la connaissance de la justice (procureur de la République), des investigations sont généralement ordonnées par le juge des enfants afin de mieux évaluer les mesures éducatives à mettre en place. Dans l'ensemble des documents à notre disposition nous nous sommes centrées ici sur les écrits qui, en amont, visent à faire connaître une situation de danger. Parmi ces écrits, une distinction doit être établie; en effet les informations sur une situation de maltraitance peuvent émaner de tout citoyen ayant connaissance de tels faits (voisin, grand-mère, appel anonyme au 115) ou d'institutions qui connaissent l'enfant (PMI, hôpital ou services sociaux scolaires ou de secteur). Parallèlement les «signalements » effectués par les services sociaux comportent d'emblée une évaluation de la situation basée sur une enquête rapide auprès de la famille. La loi de 2007 vise précisément à établir une distinction nette entre «l'information préoccupante » recueillie par les services sociaux et le «signalement», écrit rédigé par des professionnels qui évaluent la réalité du danger pour transmission au Procureur ${ }^{11}$. Dans cette perspective le corpus est constitué de dossiers recueillis dans deux Conseils Généraux différents à des époques différentes : le sous-corpus 1, recueilli dans le CG A en 2002-2003-2004, le sous-corpus 2 recueilli dans le CG A en 2006 et le sous-corpus 3 recueilli dans le CG B en 2005. Notons que le département B, département pilote, a mis en place avant la loi de 2007 une «cellule signalement» chargée de recueillir les «informations préoccupantes », et applique donc la distinction entre « information préoccupante » et « signalement $»^{12}$.

\subsubsection{L'interpellation parlementaire}

Dans le cas de l'interpellation, le corpus utilisé est composé de comptes rendus de débats parlementaires dans le Moniteur Universel, entre le mois de mai $1789^{13}$ et l'année 1834 . Nous ajoutons, pour comparaison, quelques comptes rendus, par le Journal officiel, d'interpellations parlementaires sous les troisième et quatrième Républiques. La constitution de ce corpus répond à plusieurs critères, qui appellent quelques remarques :

Le choix des dates répond à un souci d'homogénéité des sources (un seul média), et à l'hypothèse que c'est dans cette période historique qu'a pu se jouer la progressive constitution du genre de discours étudié. 
Nous avons décidé d'insérer dans le corpus les comptes rendus des Etats généraux de mai 1789. Cette décision pourrait être contestée : on pourrait en effet considérer que la Révolution française (à partir de juillet 1789) constitue une rupture profonde dans les rapports politiques et donc dans les pratiques discursives qui s'y attachent ${ }^{14}$. Or, ce point de vue devrait être relativisé, dans la mesure où ces pratiques, lors des Etats généraux de 1789, puis lors des premiers débats parlementaires, héritent de formes anciennes qui influent sur l'émergence de nouveaux genres, comme nous aurons l'occasion de l'apercevoir.

L'importance du corpus (non numérisé) explique le choix de se centrer sur quelques années, avec l'étude la plus exhaustive possibles des années 1789 à 1793, puis des études partielles jusque dans les années 1830. Seront en particulier étudiées les séances du 16 septembre 1831 et du 5 mars 1834 , pendant lesquelles commencent à s'établir les normes relatives au nouveau genre institué.

\subsection{Etude sémantique de deux verbes potentiellement performatifs : interpeller et signaler ${ }^{15}$}

Les noms de genre étudiés sont deux déverbaux de verbes potentiellement performatifs dont on peut donner quelques caractéristiques sémantiques, en particulier pour ce qui concerne leurs propriétés pragmatiques ${ }^{16}$.

Signaler, employé comme performatif (je signale X à Y), est classé par Roulet (1978) parmi les assertifs. Il s'agit en effet d'un verbe qui « engage(nt) le locuteur à un degré variable à un fait, à la vérité de la proposition exprimée » (Searle, cité par Roulet, 1978, p. 441). Il suppose un allocutaire qui n'a pas eu encore connaissance de X. Si on le compare à un verbe proche, comme informer par exemple, signaler comporte une dimension de focalisation, de mise en évidence de l'objet signalé, dimension que informer ne comporte pas avec une telle intensité. Précisons enfin que l'emploi performatif de signaler ne suppose pas nécessairement de faire agir l'allocutaire.

En ce qui concerne interpeller employé comme performatif, il peut être rapproché à la fois de l'acte d'adresse et de la classe des directifs, en particulier de la demande de faire, dont la condition essentielle, pour Searle, « revient à essayer d'amener A à effectuer $\mathrm{C} »$. En effet, interpeller revient pour le locuteur à exprimer à la fois le désir que l'allocutaire le prenne en compte dans son champ perceptif ${ }^{17}$ et qu'il interrompe son action en cours ${ }^{18}$. Ajoutons enfin que la réalisation de ce désir n'est pas forcément favorable à l'allocutaire et que l'intensité illocutoire, entendue comme degré d'intensité de l'acte, est forte.

Dans nos corpus, les déverbaux étudiés comportent des spécifications par rapport au verbe employé comme performatif, comme le remarque Dolinine (1999) à propos des noms de genre formés sur un verbe :

\footnotetext{
Aucune des typologies d'AL [actes de langage] ne tient compte des rôles des communicants, de leur statut socio-psychologique, de la situation référentielle, du canal utilisé, du temps, du lieu et des circonstances de la communication - autant de facteurs qui, en revanche, déterminent le genre. La notion d'AL qui s'appuie sur les verbes locutifs présuppose un niveau plus élevé d'abstraction et, par conséquent, une échelle typologique différente. (1999:34)
}

C'est cette relation entre la dénomination du genre et le performatif explicite que nous nous proposons d'étudier ici. Seules les conclusions de nos analyses pourront être présentées dans le cadre du format demandé.

\subsection{Etude discursive des déverbaux interpellation et signalement}

L'étude sémantique doit être accompagnée d'une étude discursive des deux déverbaux apparaissant dans des textes relevant de sphères d'activité différentes, la sphère politique pour l'interpellation et la sphère sociale pour le signalement. 


\subsubsection{Interpellation}

Des occurrences de « $\mathrm{j}$ 'interpelle» et «interpellation» apparaissent dans une période antérieure au corpus étudié, sans qu'on puisse dire si elles sont liées à un usage politique.

Les premières occurrences du corpus sont liées à l'émergence d'une évolution des conditions et des rapports politiques : la nation, s'exprimant à l'Assemblée par ses représentants, acquiert un pouvoir de plus en plus important aux côtés du pouvoir royal : de 1789 à 1791, sous un régime de monarchie constitutionnelle, puis à partir de 1792, de manière plus radicale encore, avec la proclamation de la République. Pour Guilhaumou (1989), l'acte de demande, tel qu'il va émerger dans les cahiers de doléances puis dans les débats parlementaires, constitue l'expression principale de la souveraineté du peuple. Si cette assimilation entre l'acte de demande et l'avènement des droits du peuple nous apparait exagérée $^{19}$, les verbes utilisés pour rendre compte de ce type d'acte, comme ceux qui expriment la sollicitation d'une interlocution directe, reflètent sans doute une évolution des rapports entre différentes entités politiques, soit constituées (pouvoir royal), soit en émergence (la nation).

Dans notre corpus, il est intéressant de constater que l'usage du performatif « j'interpelle», comme le nom « interpellation », dans le sens de la nomination de l'acte, sont extrêmement rares dans les premières années. Bien sûr, il n'est pas question non plus d'un usage du nom pour désigner un genre. Plusieurs verbes sont utilisés pour formuler explicitement l'acte de demande («je demande », « je propose de ») ou l'acte de sollicitation d'une interaction directe. Le genre de discours institué pour ce type d'acte, par les locuteurs comme par les scripteurs, est celui de l'adresse, c'est-à-dire d'un genre appartenant aux pratiques discursives de l'ancien régime. La fonction de ce genre ${ }^{20}$ est celle d'un message direct adressé au roi par une assemblée. Il rend ainsi compte d'un destinataire (le roi) et d'un type de rapports (les sujets à l'égard du roi). Dans notre corpus, les adresses (en tant que genre) sont parfois formulées par l'Assemblée au roi $^{21}$, mais il est intéressant de constater que le nom de ce genre est utilisé pour des messages de groupes, ou encore de députés, à destination de l'Assemblée. On peut supposer que se manifestent ainsi des types de rapports en transition, soit par changement d'allocutaire, soit par modification de la teneur même du message.

Les utilisations du performatif «j'interpelle» ou du déverbal « interpellation» sont plus nombreuses à partir de 1792 , c'est-à-dire à partir de la proclamation de la République ${ }^{22}$. Le sens du performatif peut être celui d'une adresse, d'une demande de prise en compte du locuteur. C'est le cas par exemple lors de la séance du $1^{\text {er }}$ avril 1793, lorsque Gensonné, accusé de complicité de trahison avec Dumouriez, s'adresse à Danton:

Marat.- Il a vu les lettres de Gensonné... C'est Gensonné qui était en relation intime avec Dumouriez

Gensonné.- Danton, j'interpelle votre bonne foi. Vous avez dit avoir vu la minute de mes lettres, dites ce qu'elles contenaient.

Il s'agit ici d'une adresse, renforcée par l'apostrophe, qui a pour fonction d'interrompre l'allocutaire dans son mouvement discursif et d'attirer son attention sur un objet présenté à son esprit. Les occurrences de «j'interpelle » s'accompagnent généralement d'une demande de dire, plutôt impérieuse, en adéquation avec l'intensité illocutoire propre au verbe dans un emploi performatif. Remarquons que deux constructions sont possibles: «j'interpelle $\mathrm{x}$ de [dire si/que] » équivaut explicitement à un ordre ou une question :

Danton.- ...J'interpelle le ministre de dire, si depuis la révolution, je ne l'ai pas invité à apaiser toutes les haines. (séance du 27 mai 1793)

On trouve également la construction «je vous interpelle», qui cumule l'adresse et l'annonce d'une demande :

Danton.- ...Je vous interpelle tous. (Se tournant vers les membres de la partie gauche.) Est-ce la terreur, est-ce l'envie d'avoir un roi qui vous a fait proscrire le tyran? (séance du $1^{\mathrm{er}}$ avril 1793) 
Dans ce type d'occurrences, « $\mathrm{j}$ 'interpelle » opère une forme de spécification: la valeur illocutoire directive intègre une demande de dire que ne contient pas forcément la condition essentielle de l'acception performative. Même si cette spécification ne concerne pas seulement, loin de là, les occurrences dans les activités discursives parlementaires, elle est cependant à noter.

En ce qui concerne le nom « interpellation », il équivaut dans tous les cas, à l'évaluation par le locuteur, l'allocutaire ou un tiers, d'une activité discursive dont le but pragmatique est d'interpeller. Ce sens est à contraster avec celui qu'il acquiert sous la Monarchie de Juillet. Dans la mesure où l'activité discursive d'interpeller devient à cette époque, en séance, l'objet de discussions portant sur les normes à adopter concernant cet acte, il prend peu à peu le sens de genre de discours. La séance du 16 septembre 1831 correspond à l'un de ces premiers moments d'institutionnalisation. Le député Mauguin parvient à prendre la parole en interrompant l'ordre du jour. Il évoque la possibilité d' " adresser des interpellations » aux ministres, dans un cadre qui serait accepté par l'institution :

\begin{abstract}
Je demande à la chambre la permission de lui indiquer ce que les circonstances rendent nécessaire, et d'examiner avec elle la situation nouvelle où nous allons nous trouver. Je voulais donc la prévenir que, si elle n'y mettait point d'obstacle, mon intention était de demander aux ministres des renseignements sur ce qui vient de se passer dans la malheureuse Pologne et de leur adresser des interpellations sur leur conduite dans la question belge. Nous avons, l'année dernière, admis plusieurs précédents. Un de ces précédents, c'est de ne jamais amener une discussion à l'improviste, afin de permettre à chacun de se préparer. Ce précédent est loyal, conforme aux règles parlementaires, et je ne crois pas que la Chambre veuille et doive s'en écarter. Je ne m'en écarterai pas davantage, et je demanderai aux ministres s'il leur convient que des interpellations leur soient adressées demain ou lundi.
\end{abstract}

Le sens de l'acte, dans son discours, équivaut à une adresse et une demande d'explication (demande de renseignements de la Chambre sur la Pologne et la Belgique), mais cette adresse est formulée comme une demande de droit du député à interrompre et bouleverser l'ordre du jour de manière instituée, en adéquation « aux règles parlementaires » : il s'agit en particulier de réserver un moment prévu pour les interpellations «afin de permettre à chacun de se préparer ». De plus, l'intervention de Mauguin précise l'allocutaire (les ministres, c'est-à-dire le pouvoir exécutif). Ces normes naissantes contribuent à faire émerger l'interpellation comme genre de discours ${ }^{23}$. Remarquons que se poursuit la spécification du sens politique du terme, par une restriction de l'allocutaire.

L'institutionnalisation de l'interpellation comme genre de discours va se poursuivre et se stabiliser sous les troisième et quatrième Républiques. Quelques exemples mettront en évidence cette stabilisation des normes. Tout d'abord, les interpellations sont inscrites à l'ordre du jour et sont catégorisées comme telles par l'institution. Voici comment elles sont annoncées dans le compte rendu du Journal officiel (séance du 21 janvier 1932) :

Suite de la discussion de plusieurs interpellations sur la politique générale du Gouvernement.

M. Le Président. L'ordre du jour appelle la suite de la discussion des interpellations [...] La parole est à M. Nogaro pour développer son interpellation.

Ce que le Président (et à travers lui l'institution) accorde au député, ce n'est plus exactement la permission de prendre la parole pour demander des explications au ministre, c'est le droit de produire un discours relevant d'un genre dont les règles sont connues de tous. Cela ne signifie pas que ces règles ne puissent être parfois transgressées. C'est le cas par exemple lors de la même séance, lors de l'interpellation de Jean Autrand. Le Président rappelle le député plusieurs fois à l'ordre :

M. le Président. C'est la première fois qu'un orateur prononce tout un discours en interpellant une partie de l'Assemblée [...] vous savez du reste que c'est contraire au Règlement.

M. Georges Scapini. M. Autrand interpelle sur une certaine politique. C'est son droit. 
M. le Président. Pas du tout. Il a la parole pour interpeller le Gouvernement et non une partie de l'Assemblée.

Il ajoutera quelques instants plus tard, devant une nouvelle entorse au Règlement :

M. le Président. N'oublions pas que la chambre discute des interpellations au

Gouvernement. (Rires et applaudissements à l'extrême gauche et à gauche).

Il y a certes négociations sur les normes relatives au genre de discours, mais celles-ci sont intégrées de manière forte par l'institution (inscription au Règlement, connaissances coutumières des différents acteurs, etc). Il est intéressant de noter, comme le remarque de manière très pertinente Dolinine, que le genre de discours de l'interpellation s'accompagne d'une détermination «interpellation au Gouvernement », qui permet de séparer clairement le genre de l'activité discursive et du verbe de parole. Comme le remarque avec justesse Dolinine :

[...] ce n'est pas par hasard que les dénominations de GD [genres de discours] dérivées de verbe de parole s'accompagnent toujours, ou presque, d'un déterminatif : « ordre du jour », « garantie bancaire », « interrogatoire de police », etc. (1999: 34).

Il en est de même, on va le voir, pour le genre « signalement d'enfant en danger ».

\subsubsection{Signalement}

La mise en place du signalement d'enfant en danger comme pratique professionnelle des travailleurs sociaux s'actualisant dans un écrit dont la présentation, le contenu, la réalisation même font l'objet d'une normalisation relativement poussée (guides de signalement comportant des consignes de rédaction, lesquelles peuvent être également transmises par les chefs de service) s'inscrit dans la problématique de l'émergence de la maltraitance depuis les années 1970, émergence qui se situe sur le plan juridique, mais aussi politique et médiatique (campagnes d'information nationales, large écho donné dans la presse à des cas de maltraitance non signalés par les services sociaux $)^{24}$. Du point de vue juridique, le signalement d'enfant en danger est régi par une série d'articles qui subissent plusieurs remaniements au fil des lois visant à renforcer la protection de l'enfance, dont la dernière date de 2007 : dans le code civil, il s'agit de l'article relatif à la protection de l'enfance en danger; dans le code pénal, le signalement s'inscrit dans le cadre de l'article qui fait obligation de dénoncer les mauvais traitements infligés à des mineurs. Enfin, un article du code de l'action sociale et des familles concerne plus spécifiquement les travailleurs sociaux : inscrit par une loi de 1989, l'article 226-4 stipule que «lorsqu'un mineur est victime de mauvais traitements ou lorsqu'il est présumé l'être et qu'il est impossible d'évaluer la situation ou que la famille refuse manifestement d'accepter l'intervention du service d'aide sociale à l'enfance, le président du conseil général avise sans délai l'autorité judiciaire [...]». Comme on le voit, cette obligation de signalement de mauvais traitements entre en concurrence avec le secret professionnel : la loi de 2007 autorise explicitement le partage d'informations entre professionnels soumis au secret professionnel dans le cas de suspicion de maltraitance ${ }^{25}$.

Paradoxalement il a été souvent remarqué que le mot «signalement» dans l'acception particulière de « signalement d'enfant en danger» est pour ainsi dire absent des textes de loi : on relève une occurrence dans le code pénal, à propos de la levée du secret médical pour les médecins. La loi de 2007 ne comporte que deux occurrences du terme, dont l'une qui fait office de reprise d'un prédicat verbal («avise »). Par ailleurs, l'emploi plus fréquent de « signalement » dans une circulaire du 14 mai 1993 relative à « la nondénonciation de sévices infligés à mineurs de quinze ans » fait apparaître une équivalence discursive entre signalement et dénonciation, signaler et dénoncer, qui apparaissent dans des distributions identiques («non-dénonciation de sévices»/ « signaler ces sévices»/«non-dénonciation de mauvais traitements »/«signaler ces mauvais traitements »/«l'obligation de dénonciation »/«l'obligation de signalement »). En réalité, dans les textes de loi et particulièrement dans le code pénal, signalement est employé dans le sens le plus communément enregistré par les dictionnaires (TLF informatisé, Petit Robert, édition de 1985) de «description détaillée des caractéristiques physiques d'une personne 
permettant de l'identifier » (TLF informatisé), et de façon encore plus précise, s'applique fréquemment à des personnes « recherchées » en raison d'activités délictueuses ${ }^{26}$.

Dans ce type d'occurrences, «signalement » opère ainsi une spécification de l'acception performative de « signaler » sur deux plans : l'objet signalé est considéré comme susceptible de troubler l'ordre public, comme potentiellement dangereux et l'allocutaire est une instance/ une institution (l'institution judiciaire par exemple) qui n'a pas connaissance du trouble et qui peut intervenir pour remédier à ce trouble. Ce glissement possible de «signalement» à «dénonciation》 et de «signaler» à "dénoncer » par spécification de l'objet et de l'allocutaire peut expliquer l'ambivalence ressentie par les travailleurs sociaux dans le cas des « signalements d'enfant en danger » : il s'agit en effet d'un acte fort qui peut venir rompre la relation entretenue avec la famille en provoquant l'irruption de l'Etat dans la sphère familiale (Serre, 2009).

Nous allons maintenant passer à l'analyse du corpus en faisant porter plus particulièrement notre attention sur les dénominations données aux écrits recueillis (auto-dénominations, inscrites sur le document ou hétéro-dénominations, données par les locuteurs dans les documents d'accompagnement, lettres ou bordereaux de transmission) ainsi que sur l'expression de leur visée pragmatique, tout en prenant en considération le statut respectif des locuteurs et des allocutaires ${ }^{27}$.

On insistera pour commencer sur le fait que le corpus comprend en réalité deux types de documents :

- les documents produits par des scripteurs qui ne sont pas des travailleurs sociaux (qui n'appartiennent pas aux services sociaux) : infirmière, maire, directrice d'école, mairie de quartier. Du point de vue de leurs caractéristiques « externes », ces textes revêtent des formes variées : la plupart sont assimilables à des « lettres » (identification de l'émetteur en haut à gauche, adresse, signature), même si l'un d'entre eux comporte la mention « rapport». Ces documents se caractérisent par leur relative brièveté et leur contenu factuel. On trouve également le compte rendu d'appels téléphoniques reçus par le numéro « allô enfance maltraitée ».

- les textes produits par des scripteurs qui appartiennent aux services sociaux (assistantes sociales la plupart du temps) : ils revêtent la forme d'un rapport dont le rubriquage peut être prérempli (cas habituel dans le sous-corpus 3. Les documents émanant du cadre scolaire peuvent également suivre un modèle préétabli $^{28}$ ).

On peut maintenant observer la façon dont les locuteurs dénomment les documents produits, en nous focalisant sur la dénomination «signalement», que l'on contrastera avec les autres dénominations possibles.

La dénomination « signalement d'enfants en danger » est couramment inscrite sur les documents produits par les travailleurs sociaux dans les sous-corpus 1 et 2, recueillis, rappelons-le, dans le même service, mais à des dates différentes. Dans le sous-corpus 1 on trouve également: « rapport d'évaluation pour un signalement judiciaire ». Dans le sous-corpus 2, ces documents sont dénommés « signalement », « note de signalement» ou «rapport de signalement» dans les lettres de transmission au procureur de la République. D'autres dénominations apparaissent par ailleurs sur des documents qui semblent précéder ou compléter le signalement à proprement parler : dans le sous-corpus 1, «note concernant $\mathrm{X}$ » «note d'évaluation rapide» ou encore «note complémentaire» (laquelle complète effectivement un signalement) ou « note de situation ». Cette appellation semble intervenir dans le cas d'enfants déjà suivis par les services sociaux. Une « note » peut comporter comme objet « signalement de mineur en danger et demande de saisine du Juge pour enfants et d'une audience en urgence». Le sous-corpus 2 comprend également des « notes » qui viennent remplacer ou compléter un signalement : ainsi un document appelé «note» peut-il être dénommé alternativement «note» ou «signalement»» dans la lettre d'accompagnement et même « information à l'autorité judiciaire » dans la lettre envoyée aux parents.

Le sous-corpus 3 est celui émanant du département où la normalisation est la plus aboutie : ainsi la dénomination employée pour les écrits produits par les services sociaux est-elle de façon homogène « rapport» («rapport suite à recueil d'informations», «rapport concernant les enfants»), tout en comportant comme objet (case cochée dans un tableau prérempli) « rapport suite à ouverture procédure 
enfants signalés ». Ces documents adressés au procureur de la République pour demander l'intervention du judiciaire dans une situation familiale sont des «signalements " ${ }^{29}$ d'après les préconisations de l'Observatoire National de l'Enfance en Danger relayées par la loi de $2007^{30}$. Cette normalisation vise à opérer une distinction nette entre ces «signalements » et les «informations préoccupantes » que les services sociaux ont pour mission de recueillir et d'évaluer. Cependant on observe que l'appellation «d'information préoccupante», préconisée par l'ONED puis mise en place par la loi ${ }^{31}$, qui est reconnaissable dans le syntagme «recueil d'informations», est concurrencée par la dénomination «signalement» (ou des dénominations dérivées de signaler: "signalante » à propos d'un appel téléphonique), qui reste en vigueur. Ainsi un document portant comme dénomination " fiche de recueil d'informations » peut-il contenir ensuite les mentions : "signalement reçu le », « nom de la personne qui a reçu le signalement », « information donnée par celui qui recueille le signalement ». Cette dénomination est employée dans les rapports rédigés par les travailleurs sociaux ou dans les lettres d'accompagnement (hétéro-désignation) pour catégoriser les documents, qu'ils aient la forme de rapports ou de lettres, qui émanent de l'hôpital mais aussi d'un maire ou d'un directeur d'établissement. Le suivi de la dénomination d'une lettre envoyé par un maire est à ce titre intéressant car on peut constater le flottement entre la dénomination "officielle» de "recueil d'informations» ou d' "information» et celle, en vigueur et qui sans doute correspond à l'acte accompli, de « signalement ».

Si l'on examine ensuite la façon dont s'exprime la visée performative de ces textes, on remarque tout d'abord que la présence du performatif explicite « je signale » est relativement rare; elle se trouve essentiellement dans les textes émanant des scripteurs qui ne sont pas des travailleurs sociaux :

$$
\begin{aligned}
& \text { - «par ce courrier nous tenons à vous alerter et vous signaler que la situation de X } \\
& \text { [...]», (lettre d'un chef d'établissement au procureur, sous-corpus 1) } \\
& \text { - «je voudrais vous signaler le cas d'un jeune de ma commune» (maire à Mission } \\
& \text { Protection de l'Enfance du conseil général, sous-corpus 3) }
\end{aligned}
$$

Elle entre en concurrence avec des formulations utilisant des performatifs plus neutres :

$$
\begin{aligned}
& \text { - « c'est pourquoi je vous fais parvenir ce rapport» (lettre infirmière, sous-corpus 1) } \\
& \text { - «je tiens à porter à votre connaissance les faits suivants» (directeur école à } \\
& \text { inspectrice, sous-corpus 1). }
\end{aligned}
$$

En revanche, comme nous l'avons vu ci-dessus, « signaler », « signalement » ou « signalante » apparait plus fréquemment pour rapporter et caractériser les écrits ou les paroles dans les rapports des travailleurs sociaux :

$$
\begin{aligned}
& \text { - « l'appelante veut signaler la situation d'un enfant de } 20 \text { mois ... » (fiche d'entretien, } \\
& \text { sous-corpus 3). }
\end{aligned}
$$

De fait la visée performative de l'écrit passe dans tous les cas par la description d'une situation de danger qui s'actualise de différentes façons : représentation des paroles de l'enfant au discours direct (cas de maltraitance ou d'inceste), description physique de l'enfant (trace de coups, négligences graves), description de comportements ou narration de faits qui témoignent d'une mise en danger. Comme le dit D. Serre, dans ces textes «la description vaut signal» et les consignes de rédaction des écrits de signalement insistent lourdement sur la nécessité de la précision de la description.

Cette dimension de «constat» (titre de rubrique des rapports de signalement dans le sous-corpus 3) est par ailleurs redoublée la plupart du temps par l'expression explicite de l'existence du danger ( «X est en danger »), qui reprend la formulation des textes de loi, ainsi que par l'expression de l'état psychologique de l'enfant et éventuellement de la famille, ou encore des scripteurs, qui résulte de ce constat: «X a peur », «X est inquiet », « nous sommes inquiets (pour X) » (voir Cislaru, 2008) :

$$
\begin{aligned}
& \text { - «C est une mineure qui met constamment sa vie en danger » (sous-corpus 1) } \\
& \text { - «L'ensemble des intervenants auprès de cette famille est très inquiet quant à la } \\
& \text { maltraitance vécue par J tant sur le plan physique que psychique » (sous-corpus 1) }
\end{aligned}
$$


- « La maman et nous-mêmes sommes très inquiets de cette situation et pensons que

$\mathrm{X}$ est en danger » (sous-corpus 1 )

- «Les observations [...] sont particulièrement inquiétantes » (sous-corpus 3)

Mais la demande d'intervention peut revêtir une forme explicite, qu'elle s'exprime dans un performatif («nous demandons », « nous sollicitons ») ou passe par une modalisation déontique (« il nous semble indispensable de $»)$ :

- « Nous sollicitons une mesure de protection judiciaire pour garantir aux enfants un environnement sécurisant, favorisant leur épanouissement » (sous-corpus 3)

- «Il nous semble donc primordial qu'une mesure de protection soit prise en urgence dans l'intérêt de J et $\mathrm{K} »$ (sous-corpus 1)

L'objet de la demande peut être une audience en urgence (sous-corpus 1), la mise en place d'une mesure éducative ou simplement la saisine judiciaire (sous-corpus 3, dans lequel on trouve plus rarement la préconisation d'une mesure). L'explicitation de la « demande de faire », présente quel que soit l'émetteur dans les sous-corpus 1 et 2 , est réservée aux rapports produits par les travailleurs sociaux dans le souscorpus 3, et caractérise le « signalement » tel que défini par la loi 2007.

Or l'analyse d'un corpus comportant d'autres types de rapports éducatifs nous permet de constater que cette double dimension, de description/ évaluation (évaluation sous-tendue par une description) et de proposition d'action/ demande de faire n'est pas l'apanage des rapports de signalement: on les trouve naturellement dans les enquêtes sociales ou les rapports d'investigation ordonnés par le juge suite à un signalement, mais aussi dans les rapports d'AEMO (Action Educative en Milieu Ouvert, voir Rousseau, 2007) ou même dans les «notes de comportement » étudiées par Delcambre, 1997). De fait il s'agit là de deux pratiques qui sont historiquement celles des travailleurs sociaux, assistantes sociales ou éducateurs : la pratique de l'observation (dont Rousseau, 2007, entre autres, souligne l'ancienneté) et la pratique de l'intervention. Malgré des variations dues aux conditions de "fabrication» du document (durée de la mesure, caractère pluridisciplinaire de l'équipe chargée de l'évaluation, existence de réunions intermédiaires) ou aux usages propres à un service, ces deux dimensions correspondent à des configurations discursives caractérisables linguistiquement par un certain nombre de traits (formes de représentation du discours autre, emploi et valeur du présent, nature et statut des catégorisations, ...) ${ }^{32}$.

Les conclusions auxquelles nous aboutissons sont les suivantes.

Tout d'abord, nonobstant les distinctions indiquées ci-dessus, nous faisons l'hypothèse que des configurations discursives relativement proches parcourent les différents genres produits dans le cadre du travail social (envisagé ici uniquement sous l'aspect de la protection de l'enfance).

Ce qui caractériserait le «genre » du signalement c'est que, conformément en ceci à l'emploi performatif du verbe, il regrouperait des écrits adressés à une autorité pour l'informer d'une situation qu'elle ne connaît pas. Par rapport à l'emploi performatif de «signaler», on pourrait dire que le déverbal "signalement », dans la sphère de la protection de l'enfance, implique une spécification de la nature de l'allocutaire, qui dans tous les cas est considéré comme «susceptible de faire quelque chose » et par conséquent de la nature de l'acte accompli, qui devient un «directif» et non plus seulement un « assertif» : le signalement contient toujours une demande d'intervention. Sur le plan linguistique, l'écrit de signalement quel qu'il soit comporte une description minimale des éléments susceptibles d'indiquer une situation de danger ${ }^{33}$.

Enfin, on a vu que la normalisation enregistrée par le législateur, qui restreint la dénomination «signalement» à un écrit produit par des professionnels visant à évaluer une situation de danger et à décider de la transmission de l'information au judiciaire se heurtait à une forme de résistance puisque la dénomination «signalement» continuait à circuler pour désigner tout écrit (et plus largement toute démarche) décrivant une situation de danger adressé à un destinataire susceptible de " faire » quelque chose $^{34}$, On aurait là un exemple de « découplage » entre nom de genre institué et nomination de la visée 
pragmatique. Une nouvelle enquête auprès des services permettrait de saisir la fixation et/ ou l'évolution des dénominations génériques suite à la réforme législative récente.

\section{Conclusion}

L'étude proposée dans les deux corpus, distincts sur plusieurs points, permet néanmoins de proposer quelques conclusions communes relatives à la notion de genre. Ces conclusions demanderont à être étayées de manière plus systématique.

On observe dans les deux cas une distinction de valeurs entre le performatif, la pratique discursive et le genre de discours. On note en particulier une spécification du nom de genre par rapport à la pratique discursive et au performatif correspondant : dans les deux cas, cette spécification porte sur l'allocutaire (les ministres, l'institution judiciaire) et sur la visée pragmatique dominante (demande de faire pour le signalement, demande de dire pour l'interpellation). Cette spécification, permettant l'émergence du genre, est liée dans les deux cas à une évolution des conditions socio-historiques : évolution des rapports de forces politiques dans le cas de l'interpellation parlementaire et prise en compte accrue de la protection de l'enfant dans le cas du signalement. Les modes d'institutionnalisation sont différents (plusieurs textes législatifs pour le signalement, débats permettant une normalisation progressive du genre pour l'interpellation), mais d'autres études pourraient sans doute contribuer à recenser les modalités différentes d'émergence de normes stabilisées. Enfin, l'existence de configurations discursives communes à différents genres appartenant à la même sphère discursive (ce que montre en particulier l'étude sur le signalement), le découplage possible entre le nom de genre institué et le nom donné par les locuteurs, les hésitations même de l'institution (en synchronie, comme en diachronie) en ce qui concerne le nom de genre choisi rendent effectivement complexe la relation entre nom de genre, visée pragmatique et formes linguistiques repérables dans les textes appartenant à un même genre. Cette complexité oblige à une prise en compte des relations dynamiques entre ces trois dimensions dans la définition du genre. Elle appelle enfin d'autres études précises sur des genres rendant compte de ces processus d'émergence et d'institutionnalisation : ainsi dans la sphère sociale pourrait-on étudier dans son historicité le genre de l'enquête sociale et dans la sphère politique le genre de l'adresse et de la remontrance.

\section{Références bibliographiques}

Adam, J.-M. (2001). Linguistique textuelle. Des genres de discours. Paris : Nathan.

Austin, J.L. (2002). Quand dire, c'est faire. Paris : Le Seuil (Première édition 1962, How to do things with words, Oxford).

Bakhtine, M. (1984). Les genres de discours. Dans Esthétique de la création verbale (texte de 1952-53, publié en 1979).

Beacco, J.-C. et Petit, G. (2004). Le lexique ordinaire des noms de dire et les genres discursifs. Langages 154, 87100.

Bouquet, S. (2007). «Introduction », Linx, 56, 7-18.

Branca-Rosoff, S. (2007). Genre et activité langagière : l'exemple des tchats. Linx, 56, 127-141.

Bronckart, J.-P. (1997). Activité langagière, textes et discours. Pour un interactionnisme socio-discursif. Neuchâtel et Paris : Delachaux et Niestlé.

Cislaru, G., Pugnière-Saavedra, F., Sitri F. (sous la direction de) (2008). Analyse de discours et demande sociale. Le cas des écrits de signalement. Les carnets du Cediscor, 10. Paris : PSN.

Cislaru, G. et Sitri, F. (2009). TEXTE ET DISCOURS. Corpus, co-texte et analyse automatique du point de vue de l'analyse de discours. Corpus, 8 , Corpus de textes, textes en corpus (sous la direction de J.-M. Adam et J.-M. viprey), 85-114. 
Cislaru, G. (2008). L'intersubjectivation des émotions comme source du sens : expression et description de la peur dans les écrits de signalement. In Cislaru et al., 117-136.

Collinot, A. (2006). Le « signalement d'enfant en danger ». Une problématique de l'évènement. Linx, 52, 63- 72.

Delcambre, P. (1997). Ecriture et communications de travail. Pratiques d'écriture des éducateurs spécialisés. Villeneuve d'Ascq : Presses Universitaires du septentrion.

Desquinabo, N. (2007). Intertexte générique et interprétation des actes de parole dans un corpus d'émissions de plateaux télévisés. Corpus 6, 127-152.

Détrie, C. (2006). De la non-personne à la personne : l'apostrophe nominale. Paris : CNRS Editions.

Dolinine, C. (1999). «Quarante-cinq ans après Bakhtine», Langage et société, 87, "Types, modes et genres de discours », sous la direction de S. Branca, 25-40.

Guilhaumou, J. (2007). Langue politique et opinion publique en France au XVIII ${ }^{\mathrm{e}}$ siècle. Les Langues de spécialité : regards croisés, Synergies Italie, $N^{\circ} 3,87-93$.

Guilhaumou, J.(1989). La langue politique et la Révolution française. De l'événement à la raison linguistique. Paris: Méridiens Klinckieck.

Labbe, H. et Marcoccia, M. (2005). Communication numérique et continuité des genres : l'exemple du courrier électronique]. Texto ! [en ligne vol.X, $n^{\circ} 3$, disponible sur http://www.revue-texto.net/index.php?id=512. (consulté le 19/12/2009).

Maingueneau D. (2004a). Le Discours littéraire. Paris : A. Colin.

Maingueneau, D. (2004b). Retour sur une catégorie : le genre. In Texte et discours : catégories pour l'analyse (sous la direction de J.-M. Adam, J.-B. Grize et M.A. Bouacha), Dijon, Editions Universitaires de Dijon, 107-118.

Maingueneau, D. Typologie des genres institués. Version remaniée des pages 180-187 du Discours littéraire. Paris, A. Colin, 2004, disponible sur le site http://pagesperso-orange.fr/dominique.maingueneau/intro topic.html (consulté le 19/12/2009).

Mellet, C. (à paraître). L'interpellation parlementaire : statut illocutoire et genre de discours. Communication au colloque L'Interpellation, Paris, mai 2007.

Moirand, S. (2003). Quelles catégories linguistiques pour la mise au jour des genres de discours. Journée d'étude sur Les genres de l'oral (UMR GRIC-Lyon 2), le 18 avril 2003, actes consultables: http://gric.univ-lyon 2.fr/Equipe1/actes/journees_genres.htm

Mourlhon-Dallies, F. (2007). Communication électronique et genres de discours. Glottopol, 10,. 11-23, http://www.univ-rouen.fr/dyalang/glottopol, consulté le 19/12/2009.

Von Münchow, P. (2004). Les journaux télévisés français et allemands. Plaisir de voir ou devoir de s'informer. Paris : Presses de la Sorbonne nouvelle.

Von Münchow, P. (2007). Le genre en linguistique de discours comparative. Stabilités et instabilités séquentielles et énonciatives. Linx, 56, 109-125.

Searle, J.R. (1972). Les Actes de langage. Paris : Hermann (Première édition Speech acts, Cambridge, CUP, 1969).

Searle, JR. (1976). A classification of illocutionnary acts. Langage in society 5, 11-23.

Rastier, F. (2001). Arts et sciences du texte. Paris : Presses Universitaires de France.

Récanati, F. (1981). Les Enoncés performatifs. Paris : Minuit.

Roulet, E (1978). Essai de classement syntaxique et sémantique des verbes potentiellement performatifs en français. Cahiers de linguistique $n^{\circ} 8,437-455$.

Rousseau, P. (2007). Pratique des écrits et écriture des pratiques. La part «indicible » du métier d'éducateur. Paris : L'Harmattan.

Serre, D. (2009). Les Coulisses de l'état social. Enquête sur les signalements d'enfant en danger. Paris : Raisons d'agir. 
Viaux, J.-L. (sous la direction de) (2001). Ecrire au juge. Rapport, signalement, expertise dans l'aide à la décision judiciaire. Paris : Dunod.

${ }^{1}$ «Saussure ignore le fait qu'en dehors des formes de la langue existent aussi les formes de combinaison de ces formes, c'est-à-dire qu'il ignore les genres discursifs » (1981: 90).

${ }^{2}$ Outre la réflexion de Moirand (2003) voir Maingueneau (2004 a et b), Adam (2001), Bronckart (1997).

${ }^{3}$ Un exemple, parmi d'autres, illustre le problème méthodologique et scientifique que nous soulevons : 1'intéressante analyse de Desquinabo (2007) met en évidence l'importance de la catégorie générique dans l'interprétation des actes de parole. Néanmoins, l'auteur sélectionne pour l'étude un corpus composé de « quatre genres interlocutifs supposés (controverse, portrait, polémique, jugement) », sans que l'on sache ce qui justifie qu'on puisse les identifier comme genres. On citera inversement la démarche méthodologique de Von Munchow, 2004 et 2007, qui propose de distinguer le genre "étique », permettant de constituer le corpus à partir d'une appréhension a priori du genre qui repose en partie sur le nom du genre, et le genre « émique », défini par les caractéristiques linguistiques mises au jour par l'analyse : «En choisissant des documents relevant de ce qu'on pense être un même genre, l'on aborde le genre dans une perspective étique; l'analyse met alors à la disposition du chercheur les critères linguistiques et extralinguistiques pour définir le genre émique, concept qui permet de rejuger l'attribution étique du caractère générique à tel ensemble de documents, voire de reconsidérer l'analyse même » (2007, p. 111).

${ }^{4}$ Bronckart (1997 : 76) note le «...caractère fondamentalement historique (et adaptatif) des productions textuelles : certains genres tendent à disparaître (le récit épique) mais peuvent parfois réapparaître sous des formes partiellement différentes; certains genres se modifient (cf. l'émergence du roman polyphonique ou du nouveau roman); de nouveaux genres apparaissent (cf. le tract publicitaire); bref, les genres sont en perpétuel mouvement ».

${ }^{5}$ Citons néanmoins les travaux portant sur les genres liés à l'irruption d'internet : entre autres Labbé et Marcoccia , 2005, Mourlhon-Dallies, 2007 et Branca, 2007.

${ }^{6}$ F. Mourlhon-Dallies (2007) remarque que « les noms de genre peuvent fonctionner comme un leurre ». La sphère parlementaire offre plusieurs exemples de cette distorsion: la nomination institutionnelle des «questions aux Gouvernement » ne correspond que rarement à une visée pragmatique d'interrogation. Si la forme interrogative est usuellement utilisée, elle correspond dans la très grande majorité des cas à des assertions déguisées mais réelles.

${ }^{7}$ P. Delcambre remarque par exemple que la « note de comportement», genre qu'il étudie en détail, «peut recevoir ici ou là des appellations fort diverses : rapport de comportement, note de comportement, notice de comportement, synthèse, synthèse d'évolution, bilan, bilan et demande de renouvellement » (1997, p. 43).

${ }^{8}$ sans toutefois négliger le caractère problématique de ces noms proposés par les deux auteurs.

${ }^{9}$ Cette phase du travail sera réalisée dans les prochains mois.

${ }^{10}$ Il s'agit, dans notre cas, de la représentation des débats telle qu'elle apparaît dans le Moniteur universel. Il s'agit d'un journal français créé par Panckoucke en novembre 1789. Il ne devient organe officiel qu'en 1799, mais il remplit dès sa parution une fonction publique, dans la mesure où il répond à une volonté de propager la parole émanant des assemblées.

${ }^{11} 20$ L'article L. 226-3 est ainsi rédigé :

« Art. L. 226-3. - Le président du conseil général est chargé du recueil, du traitement et de l'évaluation, à tout moment et quelle qu'en soit l'origine, des informations préoccupantes relatives aux mineurs en danger ou qui risquent de l'être. Le représentant de l'Etat et l'autorité judiciaire lui apportent leur concours. Des protocoles sont établis à cette fin entre le président du conseil général, le représentant de l'Etat dans le département, les partenaires institutionnels concernés et l'autorité judiciaire en vue de centraliser le recueil des informations préoccupantes au sein d'une cellule de recueil, de traitement et d'évaluation de ces informations. Après évaluation, les informations individuelles font, si nécessaire, l'objet d'un signalement à l'autorité judiciaire ».

${ }^{12}$ Il faut ajouter enfin que le corpus tel que nous l'entendons ne peut être restreint, et c'est particulièrement flagrant dans le cas qui nous occupe, aux seuls écrits de signalement. Ainsi notre analyse s'appuie-t-elle également sur les textes de loi (code civil code pénal, texte de la loi de 2007) et sur un certain nombre de guides de signalement élaborés dans les départements et disponibles sur internet (sur ce point voir Cislaru et Sitri, 2009).

${ }^{13}$ Le Moniteur universel n'est en fait publié qu'à partir de novembre 1789. Les numéros entre le mois de mai et le mois de décembre ont été écrits postérieurement. 


\footnotetext{
${ }^{14}$ Cette position est défendue par Guilhaumou et s'oppose en particulier à la position de l'historien F. Furet

${ }^{15}$ Nous nous intéressons ici principalement aux conditions préliminaires et essentielles selon Searle.

${ }^{16}$ Dans le cadre de cette étude, les propriétés sémantiques et pragmatiques de ces deux verbes ne peuvent être décrites que de manière succincte. Nous renvoyons à la bibliographie pour une étude plus approfondie, en particulier du verbe interpeller.
}

${ }^{17}$ Cela suppose donc que l'allocutaire n'a pas pris encore en compte la présence du locuteur dans son champ perceptif au moment de l'interpellation (d'une certaine manière, l'allocutaire est absent au locuteur).

${ }^{18}$ Cela suppose que l'allocutaire est déjà engagé dans une action au moment de l'interpellation.

19 De nombreux genres, comme les remontrances, sous l'ancien régime, expriment également des demandes d'obtention de droits plus importants.

${ }^{20}$ Qu'il serait nécessaire d'étudier de manière plus précise. D'après A. Rey, le terme français, passé en anglais, revient en France avec un sens politique et institué en genre de discours.

${ }^{21}$ En 1789,1790 et 1791.

${ }^{22}$ Sans que l'on veuille conclure de manière trop hâtive à un effet direct du changement de régime politique sur les formes langagières en usage.

${ }^{23}$ D'autres séances ultérieures (comme par exemple celle du 5 mars 1834) participent de la même élaboration.

${ }^{24}$ Cette mise en perspective s'appuie sur le travail de D. Serre (voir Serre, 2008).

${ }^{25}$ La loi de 2007 est présentée sur le site : http://www.reforme-enfance.fr.

${ }^{26}$ C'est un emploi de ce type qui a récemment choqué dans l'affaire d'une société demandant à ses salariés de « signaler » des actes illégaux commis par des collègues. Les commentaires postés sur le site $\underline{01 \text { net }}>$ Forum de 01net $>$ Les actualités de 01net. $>$ Un fichier de dénonciation de collègues suspendu par la justice (consulté le 20 décembre 2009) assimilent couramment « signaler » et « dénoncer », comme les y invitent le titre du forum.

${ }^{27}$ Comme nous l'avons signalé plus haut, cette recherche doit être complétée par une exploration dans les archives afin de mieux saisir les conditions d'émergence du genre. Notre corpus recouvre cependant un moment charnière, celui de la mise en œuvre de la loi de 2007.

${ }^{28}$ Des modèles types de «fiches de signalement» élaborés par les inspections académiques sont disponibles sur internet.

${ }^{29}$ L'objet du document, qui apparaît dans un tableau dont il suffit de cocher une case, se présente dans les formulations suivantes: "conclusions suite à ouverture 'procédure enfants maltraités ou en difficulté' »/ « signalement (hors ' procédure enfants maltraités ou en difficulté').

${ }^{30}$ «La loi réformant la protection de l'enfance réserve le terme de signalement à la saisine du procureur de la République. Le signalement est un acte professionnel écrit présentant, après évaluation, la situation d'un enfant en danger qui nécessite une protection judiciaire. » (La cellule départementale de recueil, de traitement et d'évaluation, guide pratique disponible sur le site http://www.reforme-enfance.fr).

${ }^{31}$ Voir le premier rapport de l'ONED, disponible sur le site de cet organisme : http://oned.gouv.fr/ (consulté le $19 / 12 / 2009)$

${ }^{32}$ Un certain nombre de ces traits sont présentés dans Cislaru et al., 2008.

${ }^{33}$ La dimension descriptive fait la différence par exemple avec les «fiches de signalement» sur des sujets divers (saturnisme, grippe A) que l'on peut trouver sur internet et qui ne demandent que des informations quantitatives. Cette acception de « signalement » est celle que l'on trouve dans la presse.

${ }^{34} \mathrm{C}$ 'est cet emploi qui domine dans la presse. 